\title{
On the double-mode RR Lyrae variables of the Sculptor dwarf galaxy
}

\begin{abstract}
G. Kovács`
Konkoly Observatory, PO Box 67, 1525, Budapest, Hungary

Received 22 February 2001 / Accepted 12 June 2001

Abstract. Frequency analysis of more than 300 stars of the OGLE database on Sculptor galaxy has led to the discovery of 18 double-mode RR Lyrae (RRd) variables. This yields a $20 \%$ incidence rate for double-mode pulsation among the variables previously classified as first overtone RR Lyrae stars in this galaxy. Most of the RRd stars cover the period range of $0.47<P_{0}<0$. 49 but there are two stars with longer periods of $\approx 0$. 54 . All variables fit well in the pattern of the $P_{0} \rightarrow P_{1} / P_{0}$ diagram, spanned by the RRd stars of the Galactic globular clusters and those of the Large Magellanic Cloud (LMC). It follows from our previous investigations that the luminosities and masses of the RRd stars in Galactic globular clusters and in the LMC are almost independent of metallicity. By assuming that the Sculptor RRd variables also obey this rule, with the aid of the pulsation equations we estimate their metallicities. For most of the stars we get $[\mathrm{Fe} / \mathrm{H}] \approx-1.5$, which is the same value as that obtained from a semi-empirical method for the average metallicity of the fundamental mode (RRab) stars. Two RRd stars have considerably lower metallicities, but even those are within the range corresponding to the RRab stars. The narrower metallicity range of the RRd stars is in agreement with their observed luminosity range, which is about three times smaller than that of the RRab stars.
\end{abstract}

Key words. stars: fundamental parameters - stars: variables: RR Lyr - stars: oscillations - stars: horizontal branch - globular clusters: - galaxies: individual: Sculptor dwarf spheriodal

\section{Introduction}

The variable star content of the Sculptor dwarf spheroidal galaxy has been investigated recently as a "target of opportunity" by the OGLE project, devoted primarily to searches for microlensing events (Kaluzny et al. 1995). They found 326 stars suspected of variability, from which 134 variables were classified as fundamental mode (RRab) and 89 stars as first overtone (RRc) RR Lyrae variables. They also noted the discovery of one double-mode (RRd) star. The purpose of the present paper is to conduct a more thorough search for RRd stars in the OGLE database and study their physical properties.

In a recent series of papers we have shown that doublemode variables are very useful objects in giving fairly accurate estimates on the distance moduli of benchmark systems, such as the Magellanic Clouds (Kovács \& Walker 1999; Kovács 2000a, b; hereafter KW99, K00a and K00b, respectively). A complete parameter estimation of an RRd star requires not only the knowledge of the periods but also that of two additional parameters, such as the color index and metal abundance. Unfortunately, in the case of the present data set we have light curves available only in the $V$ band. Therefore, it is necessary also to use

\footnotetext{
* e-mail: kovacs@konkoly.hu
}

some other information, in order to estimate the allowed parameter regimes for the Sculptor variables.

This additional information comes from our earlier studies on the RRd populations in various Galactic globular clusters. In those works, multicolor photometry was available, therefore, we could obtain mass and luminosity estimates. Here we examine these estimates more closely. We will see that they exhibit marginal (if any) metallicity dependence. By adopting these masses and luminosities in the present study, and applying the pulsation equations, we give estimates on the metallicities of the Sculptor RRd variables.

\section{Frequency analysis}

The method of analysis is very similar to the one used by Alcock et al. (2000, hereafter A00). The main difference is in the calculation of the frequency spectra. Here we use a single component least squares (LS) criterion (e.g., Barning 1963) rather than the standard Fourier transform method (e.g., Deeming 1975). We opted for the LS approach because in the case of the present data set with high noise and strong aliasing, this method yields a better estimate for the peak frequency position. The difference between the two methods becomes unimportant, e.g., in 


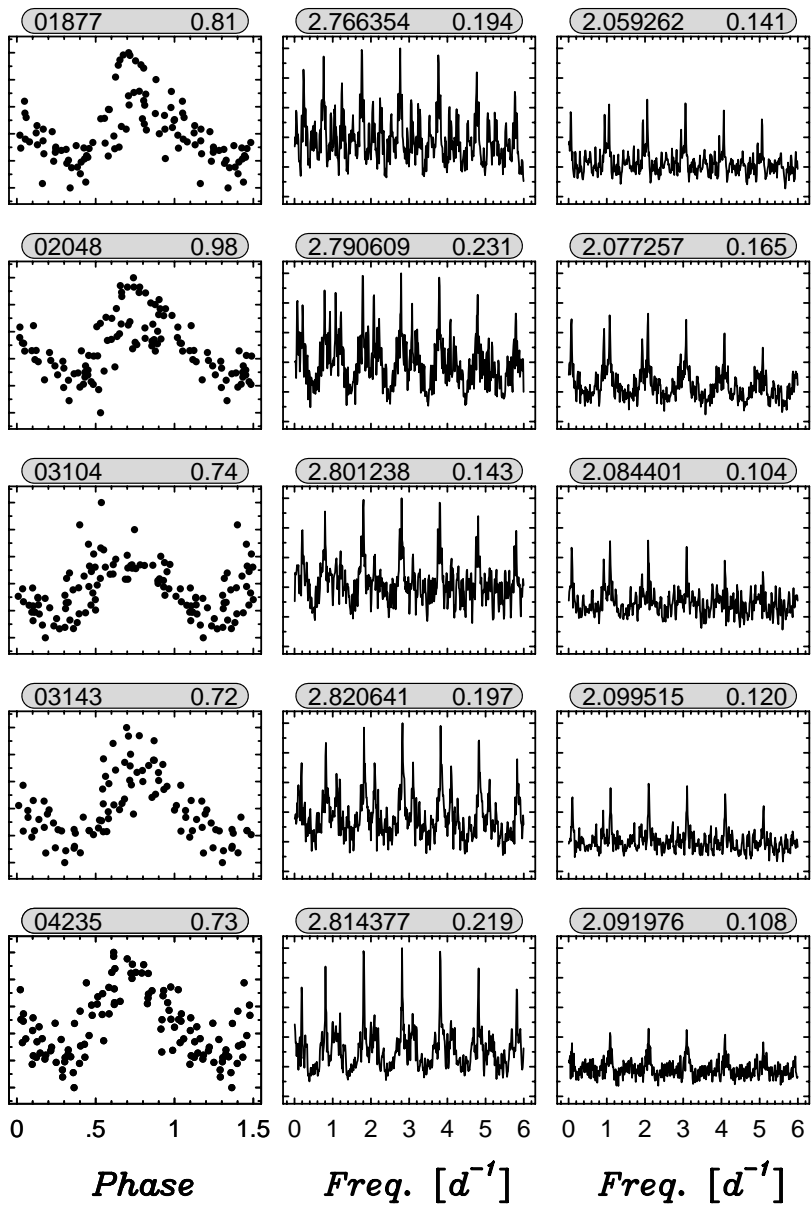

Fig. 1. Sample spectra and folded light curves for some of the most securely identified RRd variables in Sculptor. Each row displays the result of the analysis of the variable identified by the very first item of data in the respective header. First box: $V$ light curve folded with the period of the highest amplitude. Second box: LS amplitude spectrum of the original data. Third box: LS amplitude spectrum of the data prewhitened by the highest amplitude component shown in the second box. The headers show (from left to right): OGLE identifier, total range of the light variation, frequency and the corresponding amplitude for the original and prewhitened data. Amplitudes are given in [mag].

the case of RRd variable searches performed on the type of data sets analyzed by A00.

Secondary frequency components are searched by prewhitening the signal with the highest amplitude component. The full data set, containing 326 variables, is analyzed. Each time series spans some 425 days with $\approx 80$ data points in Johnson $V$ color. The data are concentrated in two observing seasons, separated by one year. The first season covers only three weeks and contains about $15 \%$ of the total number of observations. The second season covers a longer time interval, spanning of about 2.5 months. There is also a strong 1-day periodicity in the sampling, which affects the frequency spectra rather severely. However, in the present analysis, this problem is less disturbing, because nonphysical frequencies can be

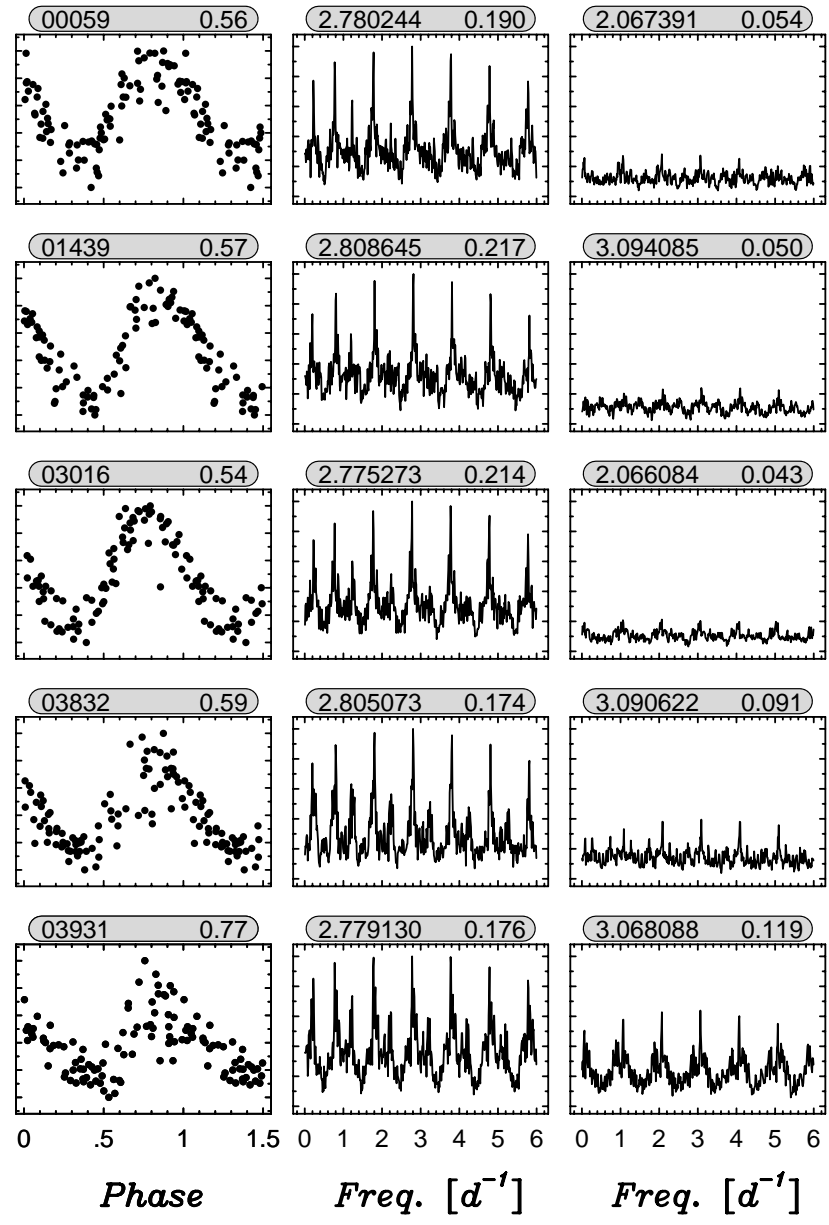

Fig. 2. Sample spectra and folded light curves of some of the less securely identified RRd variables in Sculptor. Notation is the same as in Fig. 1.

excluded relatively easily, due to the narrow period ratio range of the RRd stars $\left(0.741<P_{1} / P_{0}<0.748\right.$, if we consider all presently known RRd variables - see Popielski et al. 2000).

By visually checking all frequency spectra, 18 variables are selected as RRd candidates, based on the patterns of the prewhitened spectra. For illustration, we display some of the most secure identifications in Fig. 1. It is seen that the daily aliasing is indeed strong. Even so, the LS method is capable of identifying the correct components. (By using a standard discrete Fourier transform method, we find that three out of five stars are misidentified.) In some other cases the frequency search leads to less reliable results. Figure 2 displays some examples of this.

The lower two panels exhibit the ambiguities from the daily aliasing. In these cases physical arguments are applied to choose among the various possibilities. For example, in the case of variable \#03832, the ratio of the formally obtained frequencies is 0.908 , which excludes the possibility of both components identified as radial modes. However, the frequency difference is $0.286 d^{-1}$, which is too large for this star to be one of the newly discovered variables of RRc stars with closely spaced frequencies 
(A00; Olech et al. 1999). On the other hand, we get a very simple explanation for the observed pattern if we assume that the true secondary component is at (3.090622$1.0) d^{-1}$. This assumption leads to a period ratio of 0.7443 , which is in the right range for an RRd variable.

Table 1. Periods, amplitudes and intensity-averaged magnitudes of the RRd stars in the Sculptor dwarf galaxy.

\begin{tabular}{rcccccc}
\hline Name & $P_{0}$ & $P_{1}$ & $P_{1} / P_{0}$ & $A_{1}$ & $A_{0} / A_{1}$ & $\langle V\rangle$ \\
\hline${ }^{\star} 00059$ & 0.48370 & 0.35968 & 0.7436 & 0.190 & 0.28 & 20.16 \\
01168 & 0.54153 & 0.40357 & 0.7452 & 0.187 & 0.70 & 19.99 \\
${ }^{\star} 01439$ & 0.47809 & 0.35604 & 0.7447 & 0.217 & 0.22 & 20.16 \\
01877 & 0.48561 & 0.36149 & 0.7444 & 0.194 & 0.73 & 20.17 \\
02048 & 0.48140 & 0.35835 & 0.7444 & 0.231 & 0.71 & 20.17 \\
${ }^{\star} 03016$ & 0.48401 & 0.36033 & 0.7445 & 0.214 & 0.20 & 20.13 \\
03044 & 0.47546 & 0.35427 & 0.7451 & 0.168 & 0.58 & 20.12 \\
03104 & 0.47975 & 0.35699 & 0.7441 & 0.143 & 0.73 & 20.17 \\
03143 & 0.47630 & 0.35453 & 0.7443 & 0.197 & 0.61 & 20.20 \\
03832 & 0.47894 & 0.35650 & 0.7443 & 0.174 & 0.56 & 20.19 \\
03931 & 0.48356 & 0.35983 & 0.7441 & 0.176 & 0.68 & 20.20 \\
03941 & 0.47915 & 0.35661 & 0.7442 & 0.188 & 0.47 & 20.15 \\
04235 & 0.47802 & 0.35532 & 0.7433 & 0.219 & 0.50 & 20.22 \\
04353 & 0.48298 & 0.35983 & 0.7450 & 0.181 & 0.51 & 20.15 \\
04824 & 0.48658 & 0.36222 & 0.7444 & 0.223 & 0.46 & 20.09 \\
05354 & 0.54074 & 0.40322 & 0.7457 & 0.177 & 0.56 & 20.10 \\
05730 & 0.47254 & 0.35189 & 0.7447 & 0.180 & 0.37 & 20.20 \\
05845 & 0.48537 & 0.36135 & 0.7445 & 0.228 & 0.50 & 20.15 \\
\hline
\end{tabular}

Note: Variables labeled by $\star$ are marginal cases for RRd classification.

The other three stars in Fig. 2 have rather lowamplitude secondary components. This makes it somewhat difficult to identify them as RRd variables. The primary reason why they have been selected as probable RRd stars is that they have "correct" period ratios and their fundamental periods are sufficiently far from 0.5 . Other RRd candidates (i.e., \#00038, \#03834 and \#05015) with similarly low fundamental amplitudes have been rejected because they did not satisfy this criterion. When considering these questionable cases, we have to keep in mind that the analysis of all these variables is not yet final, because the presently available data are rather limited. Nevertheless, we do not think that considerable changes will take place in the status of most of the Sculptor RRd stars, even when more extended data become available.

The basic observed properties of the RRd variables identified in this search are listed in Table 1 (periods are in [d], the $A_{1}$ amplitudes of the first overtone components and the average magnitudes in [mag]). We note that the periods and amplitudes displayed in this table have been computed through separate LS fits, which contained the given component and its first harmonics. We see that there are only two stars with periods of $\approx 0.54$; the other variables occupy a narrow range of $0.47<P_{0}<0$. 49 . We recall that variable \#01168 was already discovered by Kaluzny et al. (1995).

From the periods alone, we may suspect that the physical properties of the Sculptor RRd variables cover similar ranges to those of the globular cluster IC 4499. Indeed, estimated from the RRab stars, the two systems have almost

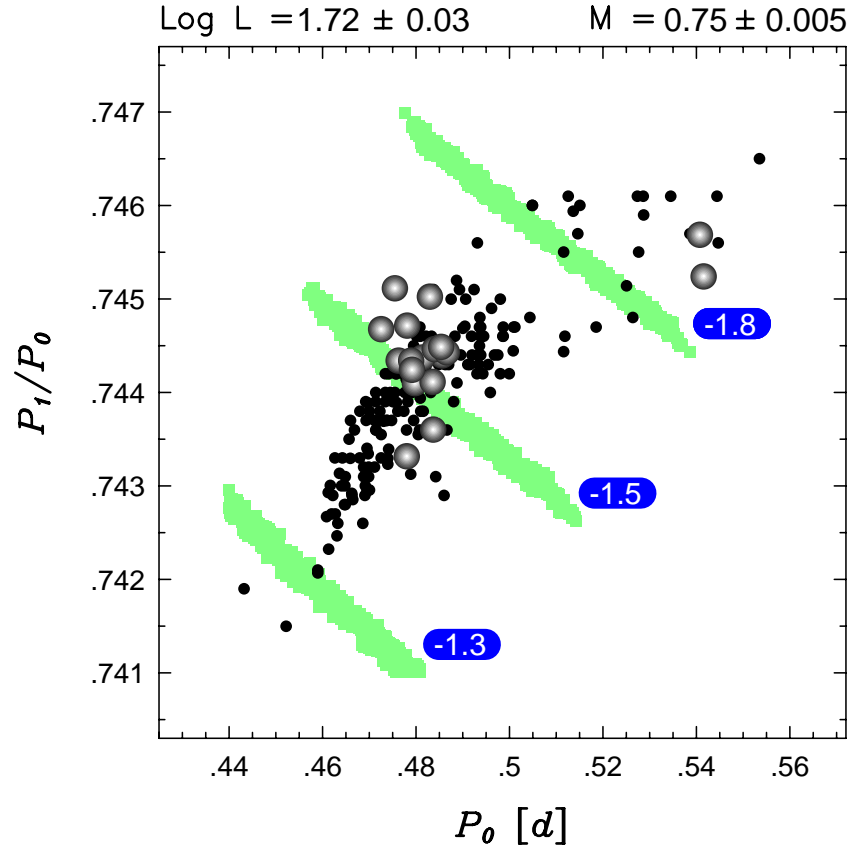

Fig. 3. Position of the Sculptor RRd variables (spheres) on the $P_{0} \rightarrow P_{1} / P_{0}$ diagram. The LMC RRd variables are plotted by dots. Shaded areas show the position of the theoretical models computed by the parameters given in the header and $[\mathrm{Fe} / \mathrm{H}]$ values displayed at the corresponding ridges. In order to place the middle of the ridges on the observed points, effective temperatures are fixed at 6900,6800 and $6700 \mathrm{~K}$ for the $[\mathrm{Fe} / \mathrm{H}]=-1.3,-1.5$ and -1.8 models, respectively. The OPAL opacities given by Iglesias \& Rogers (1996) are used with $X=0.76$.

the same average metallicity of -1.5 (see Kovács \& Walker $2001^{1}$ ). Furthermore, a display of the Sculptor RRd variables on the $P_{0} \rightarrow P_{1} / P_{0}$ diagram (often referred to as the "Petersen diagram" - see Petersen 1973), shows that they occupy a region which corresponds to this metallicity (see Fig. 3). The main factor of degeneracy in this diagram comes from the similar effects of the metallicity and mass on the period ratio. Therefore, as we will see in the next section, if no other information is available, the periods can be fitted with any metallicity between -1.3 and -2.1 , if we allow masses between 0.65 and $0.85 M_{\odot}$. It is clear that for a more reliable assessment of the physical parameters of the Sculptor RRd variables, we would need direct metallicity measurements and additional color information in order to fix the temperature and chemical composition, and thereby to provide an (almost) complete parameter input for the solution of the pulsation equations (cf., K00b). Since none of these data are available at this moment, in the next section we follow another idea, which adopts the almost metallicity-independent luminosity and mass values obtained from our former studies on RRd stars.

\footnotetext{
1 Please note that the reddened relative distance modulus $\left\langle\tilde{d}_{V}\right\rangle$ of M 4 in Table 9 of this paper is misprinted. The correct value is -4.43 .
} 
Table 2. Average stellar parameters for Galactic and LMC RRd stars.

\begin{tabular}{lccrrr}
\hline Cluster & $\mathrm{N}$ & {$[\mathrm{Fe} / \mathrm{H}]$} & $\log T_{\text {eff }}$ & $\log L / L_{\odot}$ & $M / M_{\odot}$ \\
\hline IC 4499 & 13 & -1.5 & 3.830 & 1.708 & 0.755 \\
& & & \pm 0.004 & \pm 0.017 & \pm 0.021 \\
M 15 & \multirow{2}{*}{-2.3} & 3.821 & 1.731 & 0.730 \\
& & & \pm 0.004 & \pm 0.022 & \pm 0.021 \\
M 68 & \multirow{2}{*}{11} & -2.0 & 3.820 & 1.728 & 0.756 \\
& & & \pm 0.004 & \pm 0.013 & \pm 0.016 \\
LMC & \multirow{2}{*}{181} & -1.5 & 3.834 & 1.736 & 0.778 \\
& & & \pm 0.024 & \pm 0.132 & \pm 0.100 \\
\hline
\end{tabular}

Note: Errors listed in the unlabeled rows correspond to the standard deviations of the quantities computed for the individual stars (i.e., they are not the errors of the averages).

\section{Computation of the metallicity}

In K00b we derived the distance modulus of the LMC by directly using its RRd population. The compatibility between this distance modulus and the ones obtained from the RRd stars of Galactic globular clusters and Small Magellanic Cloud Cepheids (see K00a) was also shown. Because of the consistency of all these results, we think that the physical parameters to be derived are also reliable and can be extended to the Sculptor RRd variables.

Metallicities for the Galactic globular clusters were adopted from KW99, where it was demonstrated that the condition of fixed relative distance moduli (obtained from the RRab stars with the method of Kovács \& Jurcsik 1997) yields metallicities very close to the generally accepted overall cluster values. In the case of low metallicity clusters this method does not result in a strong constraint, because of the insensitivity of the period ratios to metallicity at such low abundances. Therefore, for M 15 we took $[\mathrm{Fe} / \mathrm{H}]=-2.3$, which may be regarded as a value close to those often quoted in the literature. We will see that a somewhat higher value would be perhaps more appropriate for this cluster. For LMC we used the very recent spectroscopic data of Clementini et al. (2000) to set its average metallicity at $[\mathrm{Fe} / \mathrm{H}]=-1.5$.

Table 2 lists the average luminosity and mass values obtained in the way given in KW99 and K00b. We mention that OPAL opacities of Iglesias \& Rogers (1996) are applied with hydrogen abundance $X=0.76$ and a solartype heavy element distribution (i.e., without oxygen enhancement) in the overall heavy element content. The zero point of the color-temperature transformation is tied to the temperature scale defined by the current results of the infrared flux method (Blackwell \& Lynas-Gray 1994). For completeness, the metallicities and effective temperatures are also listed in the table. We note that this latter quantity is basically independent of the pulsation models and is determined only by the adopted color-temperature transformation.
The first systematic behavior observed from this table is the difference in the average temperature between the low- and high-metallicity variables. This temperature difference of about $150 \mathrm{~K}$ is responsible mainly for the period difference of $\approx 0$ d 05 between the low- and high-metallicity RRd stars.

By checking the mass and luminosity values, we may suspect some trends but they are rather small compared to that of the temperature. This can be seen from the very general pulsation relation (e.g., van Albada \& Baker 1973)

$$
\begin{aligned}
\log P_{0}= & 11.50+0.84 \log L / L_{\odot}-0.68 \log M / M_{\odot} \\
& -3.48 \log T_{\text {eff }}
\end{aligned}
$$

Although luminosity and mass differences may contribute to the period difference, and, in a more complicated way - not seen from the above simplified equation - to the period ratios, in the present context we assume, as a first approximation, that the masses and luminosities of the RRd stars are basically independent of metallicity.

Additional support for the assumption of the nearly constant luminosity level of the RRd stars can be lent by the narrow range of the observed average magnitudes. If we omit the the single outlier \#01168, we get 0.13 mag for the average magnitude range of the remaining $17 \mathrm{RRd}$ stars (see Table 1). On the other hand, the same quantity is about $0.4 \mathrm{mag}$ for the RRab stars (Kaluzny et al. 1995).

As far as the mass is concerned, we see that it is only M 15 that exhibits a somewhat discrepant value. By increasing the metallicity of the RRd variables of this cluster to -2.1 we get $M / M_{\odot}=0.756$ for this cluster, virtually the same value as for the other clusters. Although for this metallicity the luminosity increases to 1.747 , which is larger than the values of the other clusters, it is still within the range of our assumed \pm 0.02 ambiguity of the luminosity level in the computation of the metallicity (see later).

At this point we need to refer to the results presented by KW99. In that work we did not fix the average cluster metallicities, but they were chosen in such a way as to minimize the dispersion between the calculated and empirical relative distances (Kovács \& Jurcsik 1997). This procedure has led to increasing cluster metallicities as the metallicity of M 15 was increased. Finally, all these resulted in systematic differences between the masses of the RRd stars of the various clusters. In the interesting metallicity range for $\mathrm{M} 15$, we obtained $M_{\mathrm{RRd}}^{\mathrm{M} 68}-M_{\mathrm{RRd}}^{\mathrm{IC} 4499} \approx$ +0.02 and $M_{\mathrm{RRd}}^{\mathrm{M} 15}-M_{\mathrm{RRd}}^{\mathrm{IC} 4499} \approx-0.03$, with some sensitivity to changing chemical composition and the assumed metallicity of M 15. We think that although the optimization method of KW99 should be further studied, once even more accurate photometric and direct spectroscopic data will be available for these and other clusters, a slight difference in the photometric zero points could lead to errors in the derived relative cluster distances (see Kovács \& Walker 2001), and therefore, to an incorrect conclusion about certain intricate details, such as the mass differences between the RRd stars. 
The average parameters given in Table 2 for the LMC should be treated with caution, because the large period ratio range of its $R R d$ variables clearly indicates a substantial spread in $[\mathrm{Fe} / \mathrm{H}]$. This is probably the main reason why we get a contradictory trend in the luminosity when compared with IC 4499. Although both systems have the same average metallicity, apparently they show a considerable difference in the luminosities of their RRd stars. To clarify the situation for the LMC, we need a more thorough consideration, which might be difficult with the presently available data quality.

When considering the average masses and luminosities, two additional effects might cause some concern. As we have already mentioned in our previous papers, the zero point of the temperature scale is the main source of the systematic error in the determination of the distance modulus with the aid of double-mode variables. We note that the estimated ambiguity of $\pm 0.1 \mathrm{mag}$ in the distance modulus results in \pm 0.036 and \pm 0.018 systematic errors in $\log L$ and $M$, respectively. However, these shifts are basically independent of the metallicity, and therefore, as we will see next, do not affect our conclusion on the metallicity of the Sculptor RRd stars. The other effect on the double-mode masses and luminosities comes from a possible change in the hydrogen content. By leaving all parameters the same, but changing $X$ from 0.76 to 0.70 , we get systematic changes of +0.01 in $\log L$ and +0.024 in $M$. This effect has a stronger metallicity dependence, which yields larger differences in the average RRd masses of the various clusters (most of all, because of the relatively low adopted metallicity of M 15). Nevertheless, alike the temperature shift, this composition effect is not essential in the present rough approximation.

In conclusion, in the following we employ the constant luminosity and mass constraint to the Sculptor RRd variables to derive their individual metallicities. For $X=0.76$, we fix the average $\log L$ and $M$ values at 1.726 and 0.755 , respectively (see Table 2 ). When testing the hydrogen dependence, at $X=0.70$ the above quantities are increased as mentioned above. In order to take into account possible metallicity or other hidden dependence of these averages, we allow \pm 0.02 maximum errors both in $\log L$ and in $M$.

When the above constraints are applied to the possible parameters obtained from the observed periods, we get patterns similar to the one shown in Fig. 4. It is seen that none of the constraints, when applied alone, is able to give very useful limits on the metallicity, although the condition of constant mass yields a much stronger constraint. The very different $[\mathrm{Fe} / \mathrm{H}]$ dependence of the two constraints leads to considerable improvement in the accuracy of the metallicity determination when both constraints are applied simultaneously. Because of the assumed mass and luminosity ranges, the derived metallicities have an ambiguity of \pm 0.07 . (For the low-metallicity stars this error increases by a factor of two.) By changing the zero point of the temperature scale or using compositions with $X=0.70$, the overlapping regions given by the mass and luminosity constraints are shifted vertically

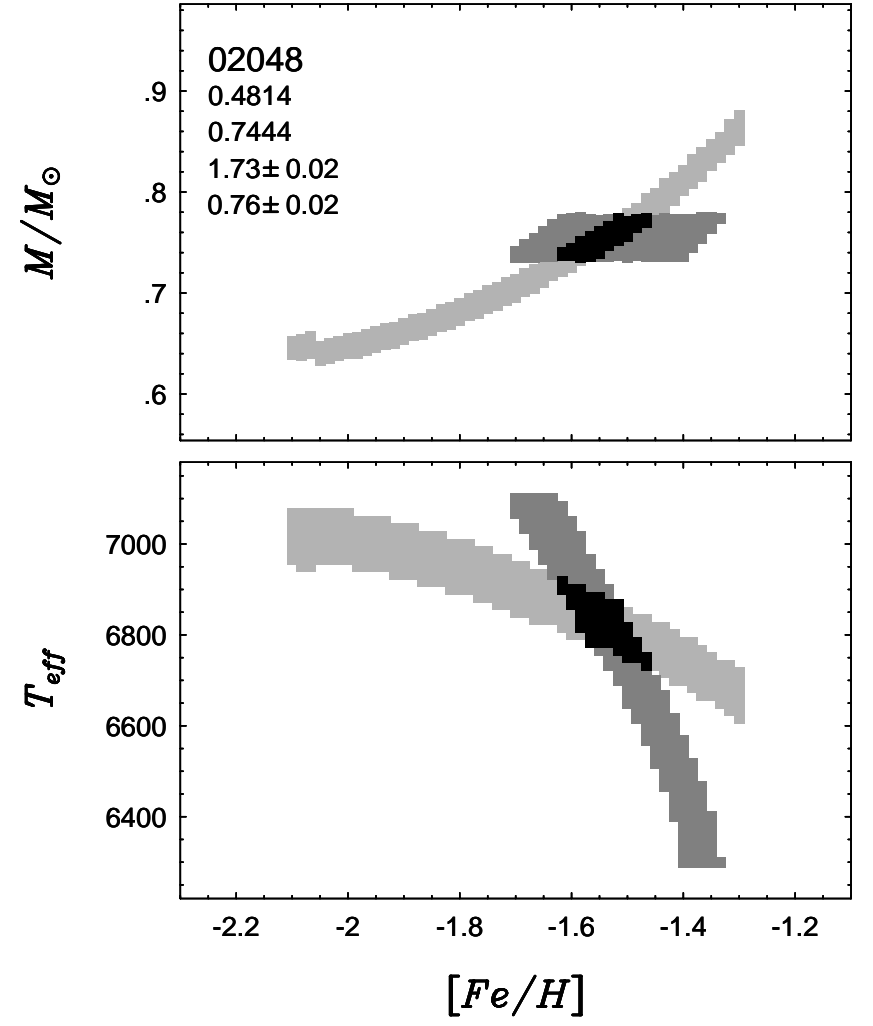

Fig. 4. Allowed parameter ranges as functions of the metal abundance. Variable name, $P_{0}, P_{1} / P_{0}$, average $\log L / L \odot$ and $M / M_{\odot}$ values and their ranges are given in the left part of the upper panel. Lightest shade corresponds to the case when only the luminosity constraint is applied, whereas the darker one shows the case when only the mass constraint is used. The darkest shade shows the parameters when both constraints are applied. Compositions with $X=0.76$ are used.

by almost the same values. This yields the same $[\mathrm{Fe} / \mathrm{H}]$ values, within 0.02 .

It is interesting to compare the metallicity distributions of the RRd and RRab stars. By using the Fourier decompositions of the light curves of the RRab stars (see Jurcsik \& Kovács 1996), Fig. 5 shows the two distribution functions. Although some part of the large scatter of the RRab metallicities might be accounted for by the large observational errors of the light curves, we think that most of the scatter is real, and reflects the chemical inhomogeneity of Sculptor. It is noticed that the $[\mathrm{Fe} / \mathrm{H}]$ range of the RRab stars is larger than that estimated from the width of the giant branch (e.g., Majewski et al. 1999, hereafter M99) and from earlier limited spectroscopic studies (Norris \& Bessel 1978). A part of this disagreement could be accounted for by the difference of the metallicity scales used in those and in our studies. We use the scale defined by high dispersion spectroscopic measurements, which is different from the more customary Zinn \& West scale (Zinn \& West 1984). If we transform the metallicity range of $[-2.2,-1.4]$ of M99, obtained from the width of the giant branch, we get ranges of $[-2.1,-1.2]$ or $[-2.3,-1.1]$, depending if we use the transformation formula of Carretta \& Gratton (1997, hereafter CG) or Jurcsik (1995, hereafter J95). Similarly, by 


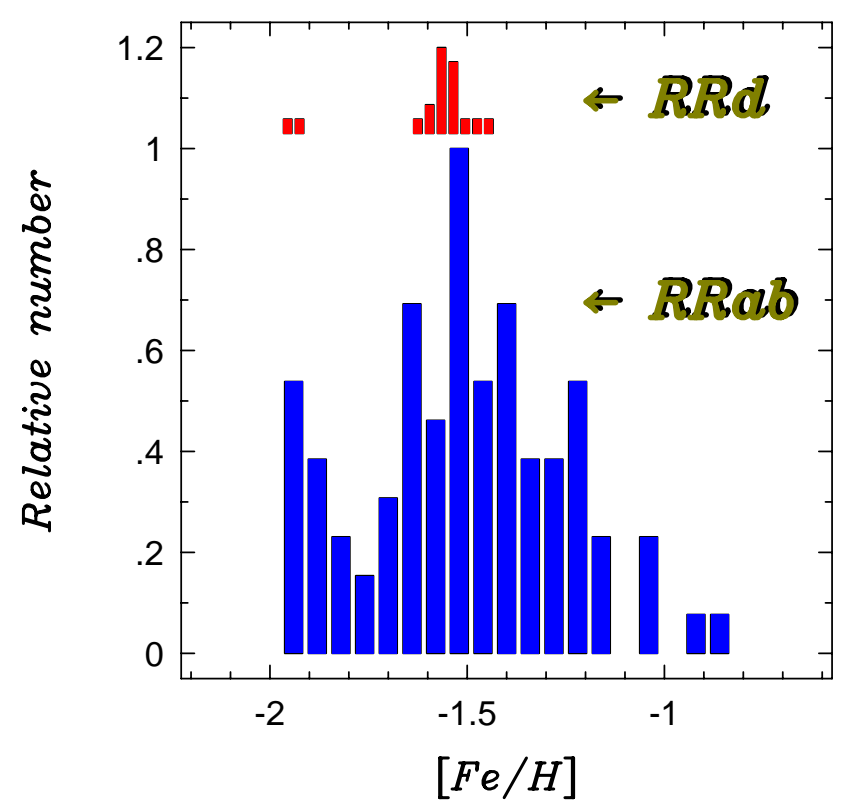

Fig. 5. Distribution of $[\mathrm{Fe} / \mathrm{H}]$ in the Sculptor among the $\mathrm{RRd}$ and RRab variables. The two distribution functions are normalized independently.

considering the result of M99 derived from evolutionary results for the Horizontal Branch stars, for the range of $[-2.3,-1.5]$ given by M99, we get ranges of $[-2.3,-1.3]$ (CG) or $[-2.4,-1.3]$ (J95). We see that although our estimate for RRab stars still yields larger $[\mathrm{Fe} / \mathrm{H}]$ values by some $\approx 0.2-0.3$ dex, the ranges indeed become similar when the same metallicity scale is used.

Most of the derived RRd metallicities are close to the average cluster value, predicted independently from the RRab stars. There are two RRd variables (\#01168 and \#05354) with considerably lower metallicities than the average. We note that these variables are among the securely identified RRd stars, and their low metallicities cannot be attributed to observational errors. The presence of low metallicity RRd stars supports the wide spread of $[\mathrm{Fe} / \mathrm{H}]$ predicted from the RRab stars.

Finally we mention two additional results. First, because of the two constraints applied, the above computation also yields effective temperatures for each variable. Without the two low-metallicity stars, for the average effective temperature of the $\mathrm{RRd}$ stars we get $\approx 6800 \mathrm{~K}$ (for the low-metallicity variables we get values lower by $\approx 200 \mathrm{~K}$ ). This can be used as a check of the assumptions made in the present derivation, when accurate multicolor photometry will be available for this system.

The second result refers to the possibility that the luminosity and mass have a slight dependence on the metallicity. By considering only the two representative clusters IC 4499 and M 68, we may suspect that the variables of M 68, which have lower metallicities than those of IC 4499, have larger luminosities (see Table 2). For the mass we may keep the assumption of metallicity-independence. If we use these constraints, we get some $50 \mathrm{~K}$ decrease in the temperature, and a very small increase of 0.05 in the metallicity for the Sculptor RRd stars. When using chemical compositions with $X=0.70$, the conclusion is very similar as far as the changes in the derived metallicity and temperature are concerned. It is noted that for this composition we get a metallicity effect in the mass, which yields higher masses for higher metallicities (the difference is $0.02 M_{\odot}$ for the above two clusters).

\section{Conclusions}

The large-scale photometric surveys connected with the search for microlensing events in various dense stellar systems, supply valuable variable star data both in amount and quality never seen before (Paczyński 2000). In this paper we utilized one of the "side products" of these projects. We analyzed the OGLE database on the Sculptor dwarf galaxy (Kaluzny et al. 1995) in order to search for doublemode RR Lyrae (RRd) stars. These variables play an important role both in the study of the simplest type of multimode pulsations, and also in the estimation of the distances of various stellar systems (Kovács 2000b).

Frequency analysis of the more than 300 variables in the OGLE database has led to the discovery of $18 \mathrm{RRd}$ stars, from which we consider at least 15 as secure identifications. As in the case of most of the RRd stars in various systems, all Sculptor variables have larger first overtone than fundamental mode amplitudes. Considering the periods, these stars are very similar to the ones found in the Galactic globular cluster IC 4499 (Walker \& Nemec 1996). Together with the two long-period stars, they also fit in the overall $P_{0}-P_{1} / P_{0}$ pattern defined by the RRd stars of the Large Magellanic Cloud (Alcock et al. 2000).

We derived individual metallicities for the Sculptor RRd variables by using the results of our former analyses on RRd stars in various stellar systems. From these works we obtained luminosity and mass values, which, in this first approximation, have been considered to be independent of the metallicity. This assumption has led to the estimation of the individual metallicities, which span the $[\mathrm{Fe} / \mathrm{H}] \approx[-2.0,-1.5]$ interval, with only two variables at the low-metallicity tail with $[\mathrm{Fe} / \mathrm{H}] \approx-1.9$. In a comparison with the independent estimation of the metallicities of the fundamental mode RR Lyrae stars (Jurcsik \& Kovács 1996), it was shown that they both lead to the same average metallicity of -1.5 . It is stressed that this conclusion does not depend on the assumed hydrogen content, effective temperature zero point and a possible slight dependence of the luminosity and mass values on the metallicity. It is also noted that our metallicities predicted from fundamental mode RR Lyrae stars are higher by $\approx 0.2-0.3 \mathrm{dex}$ than the estimates given by Majewski et al. (1999). On the other hand, when transformed to the same metallicity scale, they both yield very similar ranges of $\approx 1.0$ dex.

We recall that the analysis presented in this paper is based on the data acquired in the first phase of the OGLE project which used a $1 \mathrm{~m}$ telescope for this galaxy with the faintest RRd population known. It is clear that multicolor observation of the system with a larger aperture telescope 
would yield a unique opportunity to study the large variable star population of this galaxy. With the help of its RRd stars and other variables we could estimate its distance, study the metallicity distribution on the horizontal branch and investigate the metallicity dependence of the luminosity and mass of the RRd stars. It is also important to stress the need for direct spectroscopic observations, which, except for the first effort in this direction in the Large Magellanic Cloud by Clementini et al. (2000), are completely absent.

Acknowledgements. We are indebted to Janusz Kaluzny and his co-workers for making the Sculptor database available to us. Fruitful discussions with Béla Szeidl, Endre Zsoldos and Gáspár Bakos are appreciated. We thank the referee for his/her constructive comments. The following grants are acknowledged: ОTKA T-024022, T-026031 and $\mathrm{T}-030954$.

\section{References}

Alcock, C., et al., The MACHO collab., 2000, ApJ, 542, 257 (A00)

Barning, F. J. M. 1963, Bull. Astron. Inst. Neth., 17, 22

Blackwell, D. E., \& Lynas-Gray, A. E. 1994, A\&A, 282, 899
Carretta, E., \& Gratton, R. G. 1997, A\&AS, 121, 95

Clementini, G., Bragaglia, A., Di Fabrizio, L., Carretta, E. \& Gratton, R. G. 2000, ASP Conf. Ser., 203, 172

Deeming, T. J. 1975, Ap\&SS, 36, 137

Iglesias, C. A., \& Rogers, F. J. 1996, ApJ, 464, 943

Jurcsik, J. 1995, Acta Astron., 45, 653

Jurcsik, J., \& Kovács, G. 1996, A\&A, 312, 111

Kaluzny, J., Kubiak, M., Szymański, M., et al. 1995, A\&AS, 112,407

Kovács, G., \& Jurcsik, J. 1997, A\&A, 322, 218

Kovács, G. 2000a, A\&A, 360, L1 (K00a)

Kovács, G. 2000b, A\&A, 363, L1 (K00b)

Kovács, G., \& Walker, A. R. 1999, ApJ, 512, 271 (KW99)

Kovács, G., \& Walker, A. R. 2001, A\&A, 371, 579; erratum, A\&A, 374, 264

Majewski, S. R., Siegel, M. H., Patterson, R. J., \& Rood, R. T. 1999, ApJ, 520, L33 (M99)

Norris, J., \& Bessel, M. S. 1978, ApJ, 225, L49

Olech, A., Kaluzny, J., Thompson, I. B., et al. 1999, AJ, 118, 442

Paczyński, B. 2000, ASP Conf. Ser., 203, 9

Petersen, J. O. 1973, A\&A, 27, 89

Popielski, B. L., Dziembowski, W. A., \& Cassisi, S. 2000, Acta Astron., 50, 491

van Albada, T. S., \& Baker, N. 1973, ApJ, 185, 477

Walker, A. R., \& Nemec, J. M. 1996, AJ, 112, 2026 